National Science Foundation; the U. S. Office of Naval Research, under Grant No. N00014-81-K0438; and the U. S. National Science Foundation, under Grant No. DMR77-23776.

\footnotetext{
(a)Also at Istituto di Fisica Teorica, Università di Trieste, I-34014 Trieste, Italy.

(b) Permanent address: Institut für Physikalische Chemie, University of Vienna, Währingerstrasse 42 , A-1090 Vienna, Austria.

${ }^{1} \mathrm{P}$. Pfluger and H. J. Güntherodt, Festkörperprobleme: Advances in Solid State Physics, edited by J. Treusch (Vieweg, Braunschweig, 1981), Vol. 21, p. 271 and references therein.

${ }^{2}$ E. Wimmer, H. Krakauer, M. Weinert, and A. J. Freeman, Phys. Rev. B 24, 864 (1981).

${ }^{3}$ N. A. W. Holzwarth, S. Rabii, and L. A. Girifalco, Phys. Rev. B 18, 5190 (1978).
}

\author{
${ }^{1}$ The three systems studied in this work have $D_{6 h}$ \\ symmetry. The irreducible representations of this \\ group are labeled according to the notations of G. F. \\ Koster, J. O. Dimmock, R. G. Wheeler, and H. Statz, \\ Properties of the Thirty-Two Point Groups (MIT Press, \\ Cambridge, Mass., 1963). \\ ${ }^{5}$ According to Ref. 3, the bottom of the $\mathrm{Li}$ band in \\ $\mathrm{LiC}_{6}$ is $\sim 1.7 \mathrm{eV}$ above $E_{\mathrm{F}}$. Notice, however, that, \\ assuming rigid bands, we caloulate that $E_{\mathrm{F}}\left(\mathrm{LiC}_{6}\right)$ \\ $=E_{\mathrm{F}}\left(\mathrm{C}_{6}-\mathrm{Li}-\mathrm{C}_{6}\right)+0.4 \mathrm{eV}$ since the concentration of $\mathrm{Li}$ \\ atoms in $\mathrm{LiC}_{6}$ is twice that in $\mathrm{C}_{6}-\mathrm{Li}-\mathrm{C}_{6}$. \\ ${ }^{6}$ Observation of $\mathrm{K} 4 \mathrm{~s}$ states in $\mathrm{KC}_{8}$ at about this en- \\ ergy has been reported by J. J. Ritsko and C. F. \\ Brucker, Bull. Am. Phys. Soc. 27, 405 (1982). \\ ${ }^{7}$ L. Samuelson and I. P. Batra, J. Phys. C 13, 5105 \\ (1980), and reference therein. \\ ${ }^{8}$ W. van Haeringen and H. G. Junginger, Solid State \\ Commun. 7, 1723 (1969). \\ ${ }^{9}$ C. P. Mallet, J. Phys. C 14 , L213 (1981). \\ ${ }^{10} \mathrm{Y}$. Baer, J. Electron Spectrosc. Relat. Phenom. \\ 24, 95 (1981).
}

\title{
Localization in One-Dimensional Disordered Systems in the Presence of an Electric Field
}

\author{
C. M. Soukoulis, Jorge V. José, (a) E. N. Economou, (b) and Ping Sheng \\ Corporate Research Science Laboratories, Exxon Research and Engineering Company, \\ Linden, New Jersey 07036 \\ (Received 16 December 1982)
}

The influence of an electric field $F$ on the nature of electronic states in a one-dimensional disordered Kronig-Penney model is studied. By study of the Poincare map of the KronigPenney model in a field, the transmission coefficient $T$ was calculated as a function of system size $L . T$ is found to behave as $L^{-\alpha(F)}$, with $\alpha \sim 1 / F$, for small $F$ which indicates power-law localization. In this regime, it is predicted that the resistance $R(F) \simeq R(0)(1-b|F|)$, which may be checked experimentally.

PACS numbers: $72.10 . \mathrm{Bg}, 71.50 .+\mathrm{t}, 71.55 . \mathrm{Jv}$

The question of localization of the eigenstates in one dimensional (1D) disordered systems has been extensively studied both numerically and analytically. ${ }^{1}$ It is by now well established that in a $1 \mathrm{D}$ model all eigenstates are localized regardless of the amount of disorder. ${ }^{2}$ Experiments on quasi one-dimensional disordered metallic systems (thin wires) are in qualitative agreement with localization theory. ${ }^{3}$ While much work has been done for the study of the energy spectrum of an electron in a finite or semi-infinite periodic lattice in the presence of an electric field, ${ }^{4}$ very little is known for the problem of a 1D disordered system in an electric field, ${ }^{5,6}$ especially regarding the nature of the localized states.

It is the purpose of this Letter to examine the size dependence of the transmission coefficient
$T$ in a $1 D$ disordered system when an electric field $F$ is present. The study of the transmission coefficient has been used successfully to analyze the nature of the electronic states. ${ }^{7-10}$ The model studied in this paper is

$$
\left[-\frac{d^{2}}{d x^{2}}+\sum_{n=1}^{L} b_{n} \delta(x-n)-F x\right] \psi(x)=E \psi(x),
$$

where $b_{n}$ is the strength of the $n$th $\delta$-function potential, taken to be a random variable with rectangular probability distribution of width $W$. Here $F$ is the product of the electric field by the electron charge $e$. The numerical study of Eq. (1) can be simplified if we use the Poincare map representation of the Schrödinger equation. This consists in relating the wave-function amplitudes at different lattice sites. Specifically, defining 
$\psi_{n}=\psi\left(x=n^{+}\right)$, Bellisard et al. ${ }^{11}$ show that Eq. (1) (when $F=0$ ) can be exactly mapped to a finite difference equation of the form

$$
\psi_{n+1}+\psi_{n-1}-b_{n} \frac{\sin \sqrt{E}}{\sqrt{E}} \psi_{n}=\left(2 \cos E^{1 / 2}\right) \psi_{n} .
$$

In the periodic case, where all the $b_{n}=b_{0}$, one recovers the complete band structure of the Kronig-Penney model. In the disordered case, we recognize that Eq. (2) is formally analogous to the well studied, tight-binding Anderson model. In that case we know that all states are exponentially localized with localization length ${ }^{12}$ $l_{c} \simeq 24\left(4-E_{\mathrm{TB}}{ }^{2}\right) / W_{\mathrm{TB}}{ }^{2}$, for energies $E_{\mathrm{TB}}<2, E_{\mathrm{TB}}$ $\neq 0$, and disorder $W_{\mathrm{TB}} \lesssim 1$. If we substitute $E_{\mathrm{TB}}$ $=2 \cos \sqrt{E}$, and $W_{\mathrm{TB}}{ }^{2}=W^{2} \sin (\sqrt{E}) / E$, we get that in the Kronig-Penney model the localization length is given by

$$
l_{c} \simeq 96 E / W^{2} \text {. }
$$

Equation (3) is in good agreement with our numerical results for $l_{c}(F=0)$.

In order to map Eq. (1) to a finite second-order difference equation it is convenient to approximate the potential $F x$ by a step function. ${ }^{13}$ Once this is done, the solutions to Eq. (1) in between $\delta$ potentials are plane waves instead of Airy functions. The corresponding Poincaré map then reads

$$
\psi_{n+1}=\psi_{n}\left[\cos k_{n+1}+\frac{k_{n}}{k_{n+1}} \frac{\sin k_{n+1}}{\sin k_{n}} \cos k_{n}+b_{n} \frac{\sin k_{n+1}}{k_{n+1}}\right]-\frac{k_{n}}{k_{n+1}} \frac{\sin k_{n+1}}{\sin k_{n}} \psi_{n=1},
$$

with $k_{n}=(E+n F)^{1 / 2}$. Equation (4) reduces to Eq. (2) when we take $F=0$, as it should. This form of Eq. (1) is very useful for numerical work since it is a recursion formula and one is able to treat very long systems, being limited only by the computer time available. To carry out the iterations we can give as initial values for $\psi_{1}$ and $\psi_{2}$ the plane waves $\psi_{1}=\exp \left(-i E^{1 / 2} a\right)$ and $\psi_{2}=\exp (-2 i$ $\times E^{1 / 2} a$ ), where $E$ is the electron energy before it reaches the region where the electric field is applied, and $a$ is the lattice constant. Iterating Eq. (4) we can calculate the wave functions $\psi_{N+2}$ and $\psi_{N+3}$ to obtain the transmission coefficient from the relation

$$
T=\frac{k}{k_{1}} \frac{\left|\exp \left(2 i k_{1} a\right)-1\right|^{2}}{\left|\psi_{N+2}-\psi_{N+3} \exp \left(-i k_{1} a\right)\right|^{2}} .
$$

where $k=E^{1 / 2}, k_{1}=(E+F L)^{1 / 2}$, and $L=N a$. We have studied the random variable $\ln T$ because it is statistically well behaved, both in the $F=0$ case $^{8-10}$ and in the $F \neq 0$ case. $^{6}$ We calculated $\ln T$ for rather large systems up to $L=5 \times 10^{3}$ and ensemble averages with $1 \times 10^{3}$ members in order to obtain $\ln T$ to within $1 \%-2 \%$ accuracy. This was necessary to extract the $L$ dependence and the exponents in $\ln T$.

It is seen that when $F=0$ the states are exponentially localized with $l_{c}=96 E / W^{2}$, as expected, so that the ensemble average $\langle\ln T\rangle=-2 L / l_{c}$. This result is shown in the inset of Fig. 1. At finite electric field $-\langle\ln T\rangle$ follows a straight line $2 L / l_{c}$ for small $L$, but for larger lengths is seen to bend and increase very slowly (inset, Fig. 1). This saturation occurs about the point where the electrostatic energy $F L$ is approximately equal to $E$. To find out what is the character of the states for larger $L$ we plot $-\langle\ln T\rangle$ vs $\ln L$ in Fig. 1. We find that for the values of $F$ considered, $-\langle\ln T\rangle$ vs $\ln L$ is linear with $\ln L$, therefore suggesting that $T^{\sim} L^{-\alpha(F)}$. This behavior implies that the states are weakly localized. This type of powerlaw localization is expected to be dominant whenever $F L>E$. We notice that there is an oscillatory structure in the numerical data evident at low fields. The error bars in Fig. 1 are, however, smaller than the size of the points; therefore, we believe that the oscillations are real and related to interband or Zener-type transitions.

In Fig. 2 we plot $-l_{c}\langle\ln T\rangle / 2 L$ vs $F L / E$ for all the cases examined. The results follow a uni-

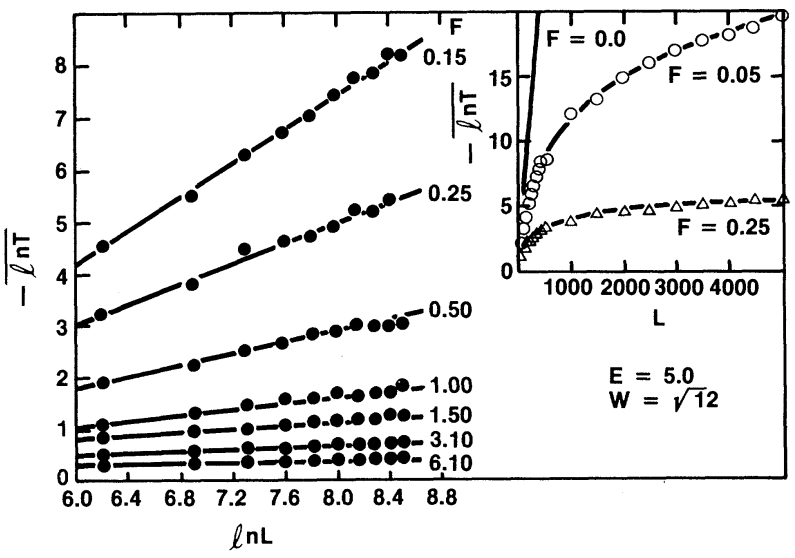

FIG. 1. Plot of $-\langle\ln T\rangle$ vs $\ln L$ for different electric fields with $E=5.0$ and $W=\sqrt{12}$. Inset: $-\ln T$ vs $\mathrm{L}$ for three different electric fields. 


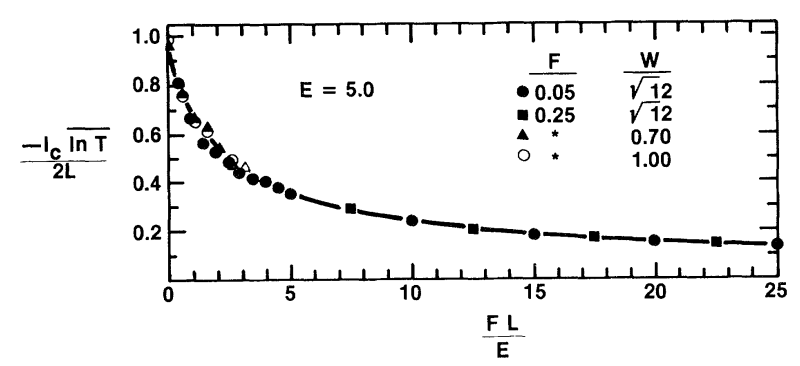

FIG. 2. Plot of $-l_{c}\langle\ln T\rangle / 2 L$ vs $F L / E$ with $E=5.0$ for different electric fields $F$ and disorder $W$. *Here $L=500$ and $F=1 \times 10^{-3}, 6 \times 10^{-3}, 1.1 \times 10^{-2}, 1.6 \times 10^{-2}$, $2.1 \times 10^{-2}, 2.6 \times 10^{-2}$, and $3.1 \times 10^{-2}$.

versal curve of the form

$$
-l_{c}\langle\ln T\rangle / 2 L=(1 / x) \ln (1+x)
$$

with $x=F L / E$. It should be pointed out that the solid curve in Fig. 2 gives an excellent representation of all the data points obtained for different $F, W$, and $L$. Points for $x>25$ are not plotted but agreement with Eq. (6) and the data is better than $1 \%$.

The analytic form of Eq. (6) can be derived from the following physical argument. When $F$ $=0$ we know that $\ln T=-2 L / l_{c}(E)$, with $l_{c}(E)$ given in Eq. (3). In the case where $F \neq 0$, we notice that the term $F x$ in Eq. (1) changes very slowly for small distances. In this case $l_{c}$ becomes an explicit function of $x$. In the spirit of an adiabatic approximation we can replace $E \rightarrow E+F x$ in Eq. (3) and take a coarse-grain average over a distance $L$. Specifically,

$$
\ln T(L) \equiv \int_{0}^{L} \ln T(x) d x=-2 \int_{0}^{L} d x / l_{c}(E+F x) .
$$

Doing the integration we recover the result given in Eq. (6). Notice that this result is inherently related to the specific $E$ dependence of $l_{c}$, and therefore essential in leading to the power-law localization given in Eq. (6). If on the other hand $l_{c}(E) \sim E^{\nu}$ with $\nu>1, T(L) \rightarrow$ const $\neq 0$ as $L \rightarrow \infty$.

In the large $-x$ limit Eq. (6) leads to the result $T=\beta(F) L^{-\alpha(F)}$ with $\alpha=+2 E / l_{c} F$ and $\ln \beta=-[2 E /$ $\left.l_{c} F\right] \ln (F / E)$. In Fig. 3 the numerically calculated values of $\alpha$ vs $1 / F$ are plotted. Note that all the points fall on a line that passes through zero. The solid line in Fig. 3 is the theoretical prediction and agrees well with the numerical data. This seems to imply that there is no electric field that makes the exponent $\alpha$ equal to zero. One could, however, define a critical field $F_{c}{ }^{(1)}$ from the relation $\alpha\left(F_{c}{ }^{(1)}\right)=1$; i.e., when $T \sim 1 / L$ the conductivity, which is proportional to $T L$, becomes independent of $L$. The relation $T \sim 1 / L$

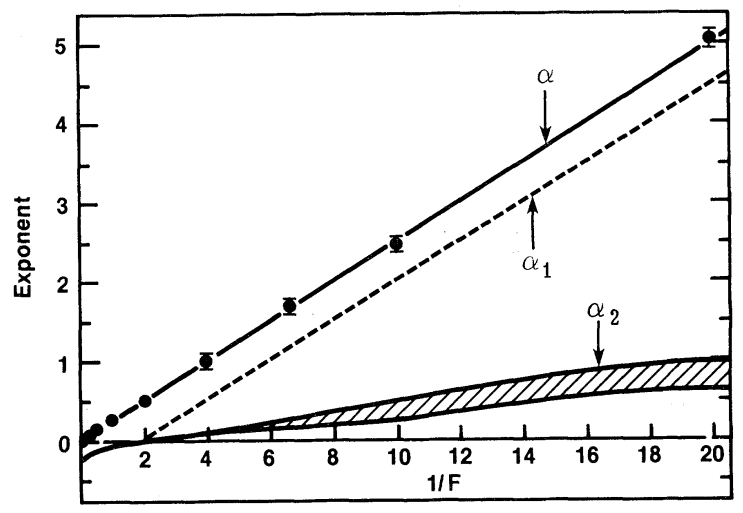

FIG. 3. Plot of various exponents vs $1 / F$. The solid circles represent the results for the exponent $\alpha$ of $T$. The solid line is the theoretical prediction $2 E / l_{c} F$. The dashed line is simply related to $\alpha$ by $\alpha_{1}=\alpha-0.5$. The hatched area represents the exponent $\alpha_{2}$ for the wave function.

is found, for example, in systems with extended states and random phases in the absence of a field. From Fig. 3 we find that $F^{(1)}=0.25 .{ }^{14} \mathrm{De}-$ fining $\alpha_{1}$ as the exponent on the right-hand side of Eq. (5), without the $k / k_{1}$ factor, we obtain $\alpha=\alpha_{1}$ +0.5 . Since $\alpha_{1}$ gives the decay of the wave function, another critical field $F_{c}{ }^{(2)}$ can be defined from the relation $\alpha_{1}\left(F_{c}{ }^{(2)}\right)=0$. As can be seen from Fig. $3, F_{c}{ }^{(2)}=0.5$. Another check on the value of $F_{c}{ }^{(2)}$ can be obtained by studying the behavior of $\psi(L)$ at site $L$. If we define $\psi(L) \sim L^{\alpha_{2}}$, then the values of $\alpha_{2}$ lie in the hatched area of Fig. 3. It is found that for small $F, \alpha_{2}$ depends on $L$, but when $F$ is large $\alpha_{2}$ is $L$ independent. It is seen that the field at which $\alpha_{2}=0$ is $F_{c}{ }^{(2)}$, i.e., the same $F$ at which $\alpha_{1}=0$. Prigodin ${ }^{5}$ defines a critical field as that when $\psi$ ceases to be normalizable. It is difficult to calculate numerically the exact eigenfunctions and to get an exponent for their length dependence. Nonetheless, from $\psi(L) \sim L^{\alpha_{2}}$, in order to have a $\psi$ normalizable we must have $\alpha_{2} \geqslant 0.5$, which implies $F_{c}{ }^{(3)}$ $\cong 0.08$. Therefore there is no unique definition of the critical field. In fact, the critical field can be quite different for different definitions. All of our numerical results do not show any sudden change of the wave function at a particular field strength.

We now turn to the interesting question of finding the resistance variation as a function of the electric field. In the case of zero electric field it was found that the resistance $R_{0}$ satisfies the relation $R_{0}=\left(\pi \pi / e^{2}\right)(1 / T-1)$. Trying to define a resistance for arbitrary field is of course not 
easy. However, it is clear that the reflection and transmission coefficients are well defined quantities for arbitrary field $F$. We can then define formally a resistance for arbitrary $F$ as $R(F) \sim[1 / T(F)-1]$ which should be right in the small-F limit. Following the scaling arguments of Anderson $e$ t al., ${ }^{8}$ it can be seen easily that the law of composition of two blocks with resistances $R_{1}(F)$ and $R_{2}(F)$ satisfies the equation $R(F)=R_{1}(F)$ $+R_{2}(F)+2 R_{1}(F) R_{2}(F)$. This leads to the result that $\ln [1+R(F)]=\ln T(F)$ is the right quantity to take averages over. Calculating this average analytically is not trivial. However, from our numerical results we have obtained

$$
\langle\ln [1+R(F)]\rangle=\left(2 L / l_{c} x\right) \ln (1+x) .
$$

In the limit when $F$ is small and $l_{c} \gg L$ we can expand the above result to get

$$
R(F)=R_{0}\left(1-\frac{L}{2 E}|F|+\frac{L^{3}}{4 E^{2} l_{c}} F^{2}+\ldots\right) .
$$

In terms of the current $I$, we can make the substitution $F=\left(\rho_{0} / A\right) I$, where $\rho_{0}$ is the residual resistivity for $F=0$ and $A$ is the area of the wire. From this result we predict that the first-order correction to the deviation from Ohmic behavior of $R$ is linear in the current and not quadratic as the standard Joule heating predicts. This behavior can be seen experimentally by measuring the resistance at very low currents and temperatures in thin wires. ${ }^{3}$

C. M. S. wants to thank G. Grest, M. H. Cohen, and L. A. Turkevitch for useful discussions. J. V. J. thanks J. Flores and G. Monsivais for many useful discussions. This work was supported in part by the National Science Foundation under Grant No. DMR-8114848.

\footnotetext{
(a)Permanent address: Physics Department, Northeastern University, Boston, Mass. 02115.
}

(b) Permanent address: Department of Physics, University of Crete, Iraklion, Crete, Greece.

${ }^{1}$ K. Ishii, Prog. Theor. Phys., Suppl. 53, 77 (1973); P. Erdös and R. C. Herndon, Adv. Phys. 31, 65 (1982), and references therein.

${ }^{2}$ H. Kunz and B. Soulliard, Commun. Math. Phys. 78 , 201 (1980).

${ }^{3}$ J. T. Masden and N. Giordano, Phys. Rev. Lett. 49 , 819 (1982); N. Giordano, Phys. Rev. B 22,5635 (1980), and references therein.

${ }^{4}$ G. H. Wannier, Phys. Rev. 117, 432 (1960); J. Zak, Phys. Rev. Lett. 20, 1477 (1968); J. R. Banavar and D. Coon, Phys. Rev. B 17, 3744 (1978); S. Nagai and J. Kondo, J. Phys. Soc. Jpn. 49, 1255 (1980).

${ }^{5}$ V. N. Prigodin, Zh。Eksp. Teor. Fiz. 79, 2338 (1980) [ Sov。 Phys. JETP 52, 1185 (1980)].

${ }^{6} \mathrm{~J}$. Flores, J. V. José, and G. Monsivais, Bull. Am. Phys. Soc. 27, 369 (1982), and to be published.

${ }^{7}$ R. Landauer, Philos. Mag. 21, 863 (1970).

${ }^{8}$ P. W. Anderson, D. J. Thouless, E. Abrahams, and D. Fisher, Phys. Rev. B 22, 3519 (1980).

${ }^{9}$ E. N. Economou and C. M. Soukoulis, Phys. Rev. Lett. 46, 618 (1981), and 47, 973 (1981), and Solid

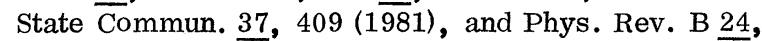
5698 (1981).

${ }^{10}$ B. S. Andereck and E. Abrahams, J. Phys. C 13 , L383 (1980); V. I. Melnikov, Pis'ma Zh. Eksp. Teor. Fiz. 32, 244 (1980) [JETP Lett. 32, 225 (1980)].

${ }^{11} \mathrm{~J}$. Bellisard, A. Formoso, R. Lima, and D. Testard, Phys. Rev. B 26, 3024 (1982).

${ }^{12}$ M. Kappus and F. Wegner, Z. Phys. B 45, 15 (1981).

${ }^{13}$ The replacement of an electric field potential by a steplike potential has been shown to lead to qualitatively the same results in the periodic case (Nagai and Kondo, Ref. 4). This approximation is expected to effect mainly the short-range behavior of the wave function. For the disordered case, because the localization effects are mainly felt at long distances, we do not expect any significant changes in our results due to this approximation even for large electric fields.

${ }^{14} F$ is measured in units $\hbar^{2} / 2 m a^{3}$, where $a$ is the lattice constant. Since $F=e E$, the unit of electric field $E$ is then $\hbar^{2} / 2 m e a^{3}$, which can be written as

$$
\left(a_{\mathrm{B}} / a\right)^{3} m e^{4} / \hbar^{2} 1 /\left(2 e a_{\mathrm{B}}\right)=\left(a_{\mathrm{B}} / a\right)^{3}(13.6 \mathrm{~V}) / a_{\mathrm{B}} \text {. }
$$

Here $a_{\mathrm{B}}$ is Bohr's radius. 